\title{
Immunomodulatory activity in tumor-bearing mice treated with Withania somnifera extract
}

\begin{abstract}
We investigated some actions of Withania somnifera on the growth and differentiation of hematopoietic precursors [granulocyte/macrophage colony cell formation (CFU-GM)] of normal animals and EAT bearers, which were treated with different doses $(20,50$, or $100 \mathrm{mg} / \mathrm{kg} /$ day). We also evaluated the presence of colony stimulatory factors in the animal's serum, as well as its survival. Furthermore, we analyzed lymphocyte proliferation, IFN- $\gamma$, and TNF- $\alpha$ concentrations in treated bearing mice. Our results demonstrated Withania somnifera effectiveness on hematopoietic precursors growth and differentiation in marrow and spleen TAE-bearing mice. As it was already expected, EAT produced myelosuppression and increased CFU-GM spleen number concomitantly. The treatment of EAT-bearing animals with W.S. $(20,50$, and $100 \mathrm{mg} / \mathrm{Kg})$ produced a dose-dependent increase in myelopoiesis, an increase in a lifetime, and a reduction in spleen colony number. All this happened parallel to survival. As to lymphocyte proliferation, they were also dose-dependent in treated bearing animals. Concerning IFN- $\gamma$ levels, we observed a significant reduction in non-treated bearing mice. Levels of TNF- $\alpha$ of treated bearing mice significantly increased when compared to the non-treated bearing group. These results are encouraging since they favor the use of W.S. extract in therapeutic combinations with other chemotherapeutic agents to reduce myelotoxicity and supplement the tumoricidal efficacy of this plant.
\end{abstract}

Keywords: ehrlich ascites tumor, antitumor, myelopoiesis, withania somnifera, CFU-GM
Volume 10 Issue 2 - 2021

\author{
André Luiz de Melo,' Maria Cristina \\ Marcucci, ${ }^{2}$ Claudemir de Carvalho, ${ }^{3}$ Carolina \\ Passarelli Gonçalves, ${ }^{4}$ Adriana de Melo, ${ }^{1,5}$ \\ 'Department of Pharmacy, UNIPINHAL, Regional University \\ Center, Espírito Santo do Pinhal, SP, Brazil \\ 2Postgraduate Program in Oral Science and Diagnosis, UNESP, \\ Institute of Science and Technology, São José dos Campos, SP, \\ Brazil \\ ${ }^{3}$ Dean of Research and Postgraduate Studies, University Center \\ FUNVIC, Pindamonhangaba, SP, Brazil \\ 4Postgraduate Program in Pharmacy and Biotechnology, São \\ Paulo Anhanguera University, SP, Brazil \\ ${ }^{5}$ Department of Pharmacology/Blood Center, Medical Science \\ Faculty, FCM, State University of Campinas, UNICAMP, \\ Campinas, SP, Brazil
}

\begin{abstract}
Correspondence: Adriana de Melo (Ph.D.), Department of Pharmacy, Centro Regional Universitário de Espírito Santo do Pinhal (UNIPINHAL), Avenida Hélio Vergueiro, s/n, CEP I 3990 000. Espírito Santo do Pinhal-SP, Brazil,Tel +55-19-365I-9600, Email prof.adriana.melo@unipinhal.edu.br; koymelo@yahoo.com.br
\end{abstract}

Received: March 29, 202I | Published: April 12, 202 I
Abbreviations: ADME, absorption distribution metabolism and excretion; CSFs, colony-stimulating factors; GABA, $\gamma$-aminobutyric acid; GM-CFU, bone marrow/spleen hematopoietic precursors granulocyte-macrophage series; MTT, tetrazolium reduction method; EAT, Ehrlich ascites tumour

\section{Introduction}

Ashwagandha (Withania somnifera, W. somnifera) is an ancient Ayurvedic drug that has been used to treat a variety of disorders ranging from dropsy, pulmonary tuberculosis, disorders of nervous and reproductive systems to skin lesions. It acts as an antitumor, antiarthritic, anti-inflammatory, antipyretic, analgesic, antispasmodic, and hypotensive. Ashwagandha also prevents carbon tetrachlorideinduced hepatotoxicity in mice and rats. In the quest for therapeutic agents with potent pharmacological action and minimal toxic effects, Ashwagandha has been investigated extensively over three decades. The pharmacological of the whole extract and its separate components, alkaloids, and steroidal lactones, have been studied on experimental animals and human volunteers. These studies have proven their pharmacological action with minimal toxic effects than the available allopathic drugs. ${ }^{1-4}$ Historically, $W$ somnifera has been used in Ayurvedic medicine for the treatment of skin tumors. The scientific inquiry into the efficacy of the various constituent compounds of $W$ somnifera in preventing or delaying tumorigenesis indicates differential mechanisms and efficacy. Dhuley et al. ${ }^{5}$ demonstrated that $W$. somnifera significantly inhibited ochratoxin-A induced suppression of chemotactic activity and production of interleukin-1 and tumor necrosis factor by macrophages in mice. The use of in silico methods has been an interesting strategy to accelerate the discovery of new potential drugs, or to find new targets for the biological application of already known substances. In addition, the in silico studies of the designs of potentially active structures covers, from the structureactivity relationship to toxicological and pharmacokinetic studies (ADME: absorption, distribution, metabolism and excretion).

In addition, Shohat et al. ${ }^{6}$ investigated the effect of isolated withaferin-A on the growth of Ehrlich ascites tumor cells in Swiss albino and BDF1 mice. In these studies, the analysis of cell cycle progression indicates that withaferin-A inhibits tumor growth by arresting mitosis in the metaphase. It has been suggested that additional factors, particularly immunological support, should be involved. Hence, it is of interest to evaluate the actions of $W$ somnifera on the immune system. ${ }^{7}$ Tumor growth has been reported to induce various phenotypic and functional changes in the cellular constituents of the host immune system. These changes have been implicated as mechanisms by which tumors subvert potential beneficial host responses. ${ }^{8,9}$ Among the many mechanisms that favor tumor growth, it is postulated that soluble factors generated during tumor growth can affect the amount of granulocyte-macrophage progenitors and inhibit various responses. ${ }^{10-14}$ The Ehrlich ascites tumor (EAT), a rapidly growing experimental model, has been found to induce profound alterations in the cell populations of critical importance to ensure host protection, and macrophages are in part responsible for the impaired immune response in tumor-bearing mice. ${ }^{15-18}$ Both granulocytes and macrophages are derived from a hematopoietic stem cell in the bone marrow. Quantitative measures of the hematopoietic cell proliferative capacities are examined through the colony growth of progenitor cells in vitro in the presence of colony-stimulating factors (CSFs). ${ }^{19-22}$ In vitro colony assays may be an early indicator of the hematopoietic 
changes associated with tumor growth, and also, the potential for exhaustion of the hematopoietic system during the host's life span has special significance for cancer patients. ${ }^{10,23,24}$

The anti-inflammatory potential of this plant seems to be due to the action of its withanolide components in the regulation of protein synthesis. ${ }^{2}$ Pre-clinical studies suggest efficacy in treating Alzheimer's disease, mechanism of action is attributed to its behavior as an agonist receptor of GABA ( $\gamma$-aminobutyric acid). ${ }^{25} \mathrm{~W}$. somnifera is also characterized as adaptogenic, as it relieves physical and psychological stress, with anti-inflammatory, tranquilizing, anthelmintic, aphrodisiac actions, among others. ${ }^{3,6}$ Experimental studies demonstrate a significant activity of this plant in the prevention and regression of tumorigenesis through an increase in the chemotactic activity of macrophages and the production of cytokines, such as interleukin 1 and the tumor necrosis factor- $\alpha .{ }^{5}$ In this study, the structure of compounds Withaferin-A (1), a major component of the W. somnifera extract, was considered for the in silico analysis of its physico-chemical, pharmacokinetic properties and in the determination of possible targets and thus corroborating the biological results found. Based on the above reports, as a second objective, we evaluated the effects of $W$. somnifera extract on the growth and differentiation of bone marrow/ spleen hematopoietic precursors granulocyte-macrophage series (GM$\mathrm{CFU}$ ) in the proposed experimental model. Besides, we investigated the effects of this extract on the specific response to the tumor through the cytokines produced by the subpopulations of helper $\mathrm{T}$ cells called "T helper" (Th1) -IFN- $\gamma$, and of pro-cytokine inflammatory (TNF- $\alpha$ ), the production of hematopoietic cell colony-stimulating factors. We also studied the cytotoxicity of this extract, using the tetrazolium reduction method (MTT).

\section{Material and methods}

\section{Target prediction}

The PharmMapper server is open and free access (http://www.lilabecust.cn/pharmmapper/). The file containing the optimized structure of compound 1, was converted to an input file .mol2 and inserted in the server, with the methodology Druggable Pharmacophore Models $(54-56) .^{26-28}$

\section{Pharmacokinetic properties}

To evaluate the pharmacokinetic properties of the designed compounds, the 2D structure of the compounds were drawn on Chemdraw Ultra 12.0. Each structure was imported and the structure smiley was entered at the interface of the website (http://swissadme. $\mathrm{ch} /$ ). The SwissADME drug design study was run and the ADMET properties/parameters were generated..$^{29}$

\section{Molecular electrostatic potential map}

The three-dimensional model designed for the compound was subjected to geometric optimization by molecular mechanics under the $\mathrm{MM}+$ force field and, subsequently, the calculation of partial atomic charges using the PM3 semi-empirical methodology, through the HyperChem 8.0.8 software. The file containing the three-dimensional information of the structure as well as the charge information of electrostatic potential was the input files and considered for viewing by the software JMol.

\section{Plant extract}

Dry extract of $W$. somnifera was provided as an orange medium brown powder with a slight characteristic odor, containing $1 \%$ of total alkaloids and $1.5 \%$ of withanolides, by Galena Química e Farmacêutica Ltda. (Campinas, SP, Brazil). The extract was supplied in distilled water and diluted immediately before use in appropriate concentrations.

\section{Animals and mouse tumor model}

Male BALB/c mice, 6-8 weeks old, were used for experiments. The Ehrlich ascites tumor (EAT) was used for in vivo evaluation of cytokine profile, mitogen-induced spleen cell proliferation. Animal experiments were approved by the Animal Experimentation Ethics Committee of the Faculty of Pindamonhangaba, protocol CEEA 031/2012 and Use Committee that follows the recommendations of the Canadian Council on Animal Care. Ehrlich ascites tumor was maintained in $\mathrm{BALB} / \mathrm{c}$ mice in ascites form by serial transplantation. Tumor cell suspensions were prepared in balanced salt solution at $\mathrm{pH}$ 7.4 to final concentrations of $6 \times 10^{7}$ viable cells $/ \mathrm{mL}$. In all experimental protocols described, mice were inoculated intraperitoneally (i.p.) on day zero with $6 \times 10^{6}$ viable tumor cells per mouse in a volume of 0.1 $\mathrm{mL}$. Viability, assessed by the trypan blue dye (Sigma Chemical Co., St. Louis, USA) exclusion method, was always found to be $95 \%$ or more. $W$ somnifera extract was administered orally in standardized doses, using a $0.9 \%$ saline solution as the administration vehicle. Uninoculated control animals were administered $W$ somnifera in different doses for 10 days. The inoculated and treated animals received $W$ somnifera for 10 days, and the tumor-bearing animals were inoculated $2 \mathrm{~h}$ before the start of treatment. The doses were 20 , 50 , and $100 \mathrm{mg} / \mathrm{kg}$.

\section{Drug and treatment regimen}

Doses of 20,50 , or $100 \mathrm{mg} / \mathrm{kg}$ of $W$. somnifera were administered for 10 consecutive days to groups of normal and tumor-bearing mice by gavage (i.o.) of $0.2 \mathrm{~mL}$ per mouse. The treatment started 0 days after tumor inoculation and continous for 10 days. The progenitor cell assays were performed on the first day after the last administration. Each experiment included parallel control groups of normal and tumor-bearing mice treated with an equivalent volume.

\section{Progenitor cell assay}

Assays with cell suspensions from femoral marrow and spleen were performed in $2 \mathrm{~mL}$ agar cultures in $35 \mathrm{~mm}$ Petri dishes using 1 x $10^{5}$ marrow cells or $2.5 \times 10^{5}$ spleen cells per culture. The medium used was Dulbecco's modified Eagle's medium (DMEM - Sigma Chemical Co., St. Louis, MO) containing 20\% fetal calf serum and $0.6 \%$ agar. Colony formation was stimulated by the addition of the rmGM-CSF described above. The cultures were incubated for seven days in a fully humidified atmosphere of $10 \% \mathrm{CO}_{2}$ in the air, and colony formation (clones $>50$ cells) was scored at $35 \mathrm{X}$ magnification using a dissection microscope. ${ }^{20}$

\section{Preparation of spleen cell suspensions}

Suspensions of spleen cells from all mice were prepared by gently pressing aseptically removed spleen through a stainless-steel mesh net. The cells were suspended in RPMI 1640 culture medium (Sigma Chemical Co., St. Louis, USA) supplemented with 5\% foetal calf serum (FCS) and washed twice. Red blood cells were lysed with $0.17 \mathrm{M} \mathrm{NH}_{4} \mathrm{Cl}$ and the remaining cells were again washed three times and counted. Viability was determined by trypan blue exclusion and consistently exceeded $90 \%$ 


\section{Mitogen-induced proliferation}

Splenocytes were suspended at $1 \times 10^{6}$ cells $/ \mathrm{mL}$ in RPMI 1640 supplemented with $25 \mathrm{mM}$ HEPES, $25 \mathrm{mM}$ sodium bicarbonate, $2 \times 10^{-5} \mathrm{M} 2$-mercaptoethanol, $2 \mathrm{mM}$ L-glutamine, $100 \mu \mathrm{g} / \mathrm{mL}$ streptomycin, $80 \mu \mathrm{g} / \mathrm{mL}$ gentamycin (enriched medium) and 5\% FCS (Sigma). Two hundred and fifty microliters of the cell suspensions were seeded into 96-well microtiter plates (Corning, New York, USA) in the presence of $5 \mu \mathrm{g} / \mathrm{mL}$ Con A (Sigma), and incubated at $37^{\circ} \mathrm{C}$, in $5 \% \mathrm{CO}_{2}$. After $72 \mathrm{~h}$ incubation, cultures were pulsed with $1 \mu \mathrm{Ci}\left[{ }^{3} \mathrm{H}\right]$ $\mathrm{TdR}(5 \mathrm{Ci} / \mathrm{mmol}$, Amersham, Little Chalfont, UK) for an additional period of $18 \mathrm{~h}$. The cells were then harvested and $\left[{ }^{3} \mathrm{H}\right] \mathrm{TdR}$ uptake estimated by liquid scintillation counting. Data are expressed as mean cpm $\left[{ }^{3} \mathrm{H}\right] \mathrm{TdR}$ incorporation $\pm \mathrm{SD}$ of triplicate cultures of each animal, corresponding to 8 mice/group.

\section{Induction of mouse cytokine secretion in vitro}

Splenocytes $\left(1 \times 10^{6}\right.$ cells $\left./ \mathrm{mL}\right)$ were suspended in enriched medium supplemented with 5\% FCS and seeded into 24-well culture plates (Corning) in the presence of $5 \mu \mathrm{g} / \mathrm{mL}$ Con A. Cell-free supernatants were collected after $24 \mathrm{~h}$ of incubation at $37{ }^{\circ} \mathrm{C}$, in $5 \% \mathrm{CO}_{2}$, and cytokine levels were detected by ELISA (Biotek $800 \mathrm{TS}$, Absorbance Reader, USA).

\section{Quantitation of Cytokine Levels (IFN- $\gamma$ and TNF- $\alpha$ )}

For the cytokine dosage, the splenic T-cell cultures were used for the dosage of cytokine. The cellular suspensions used in these cultures were prepared, as described previously. In the holes of the culture plate ( 24 wells) containing $800 \mu \mathrm{L}$ of RPMI-1640 medium enriched with $5 \%$ fetal bovine serum, penicillin $(100 \mu \mathrm{g})$ HEPES $25 \mathrm{mM}$, 2-mercaptoethanol $(1: 100)$ and glutamine $(216 \mathrm{mg} / \mathrm{mL})$ were added $200 \mu \mathrm{L}$ of cell suspension $\left(1 \times 10^{6}\right.$ cells $\left./ \mathrm{mL}\right)$ and $50 \mu \mathrm{L}$ Con A (final concentration: $2.5 \mu \mathrm{g} / \mathrm{mL}$ ). The plate was incubated in a humid greenhouse containing $5 \%$ of $\mathrm{CO}_{2}$ at $37^{\circ} \mathrm{C}$. After 24 and 48 $\mathrm{h}$, supernatants corresponding samples were collected and stored at $-20^{\circ} \mathrm{C}$ for the dosage of the cytokines under study. Cells from each sample were incubated in the absence of a stimulus (ConA) to verify spontaneous stimulation.

The measurement of cytokines TNF- $\alpha$ and IFN- $\gamma$ was performed by the immunoenzymatic method - Capture ELISA (Pharmingen, USA) using the following Kits (BD OptEIA ${ }^{\mathrm{TM}}$ ELISA Set): BD OptEIA $^{\text {TM }}$ ELISA Set Mouse TNF- $\alpha$ (Mono/Mono) (Catalog number 555268) and BD OptEIA ${ }^{\mathrm{TM}}$ ELISA Set Mouse IFN- $\gamma$ (Catalog number 555138). The sensitized supernatants obtained in the cultures were placed in 96-hole microplates (NUNC- Maxisorp, DM) sensitized by adding $100 \mu \mathrm{L}$ of the capture antibodies diluted in PBS buffer ( $\mathrm{pH} 7.0)$ of the respective kits and incubating overnight at room temperature. After this period, the plates were washed 4 times with PBS containing $0.05 \%$ Tween 20 (PBS/Tween), and the nonspecific reactions blocked with $200 \mu \mathrm{L}$ of PBS containing 10\% SBF (PBS/SBF) for $1 \mathrm{~h}$ at room temperature. The overnight of the other splenic cell cultures and patterns (recombinant antibodies diluted in PBS/SBF) were added in duplicate and incubated for $2 \mathrm{~h}$ at room temperature. After this period, the plates were washed 4 times with PBS/Tween, and the monoclonal anti-cytokine antibodies of mice labeled with biotin diluted in PBS/ SBF were added.

After $1 \mathrm{~h}$ of incubation at room temperature, the plates were washed 6 times with PBS/Tween $100 \mu \mathrm{L}$ of avidin-peroxidase solution (Sigma Chemical Co, USA) diluted in PBS/SBF (1:400 of a $1 \mathrm{mg} / \mathrm{mL}$ solution) was added. After 30 minutes of incubation, 100 $\mu \mathrm{L}$ of the substrate $\left(10 \mu \mathrm{L} \mathrm{H}_{2} \mathrm{O}_{2} \quad 30 \%\right.$ and $5 \mathrm{mg}$ of dihydrochloride o-phenylenediamine (Sigma) dihydrochloride were added in $10 \mathrm{~mL}$ of citrate buffer, $\mathrm{pH}$ 4.35). The o-phenylenediamine dihydrochloride is a chromogen suitable for use in ELISA protocols that use conjugates with peroxidases. Thus, the enzyme avidin-peroxidase acts on this substrate to form a soluble and brown-colored final product. The formation of the final product was read spectrophotometrically at 492 $\mathrm{nm}$ in an ELISA reader (Labsystem). Labsystem Since the relationship between the absorbance values at $492 \mathrm{~nm}$ and the normal cytokine concentrations present in the solution describes a linear function, the cytokine concentration present in the sample can be calculated. The results will be expressed in $\mathrm{pg} / \mathrm{mL}$.

\section{Realization of the survival curve}

In order to observe the possible protection of animals treated with W. somnifera, a survival curve was performed. Four experimental groups were considered: control animals and EAT carriers, animals treated with W.S. at doses of 20,100 or $100 \mathrm{mg} / \mathrm{kg}$ for 10 consecutive days, and inoculated with $6 \times 10^{6}$ cells/animal of TAE 2 hours before the beginning of treatment with $W$. somnifera.

\section{Statistical analysis}

Analysis of variance was used to evaluate the CFU-GM variables. When statistical differences between groups were detected, the Tukey contrast test was used. For evaluating the variables, IFN- $\gamma$ and TNFwere verified using Kruskal-Wallis ANOVA and Wilcoxon test $a$ posteriori. For the survival curve of the group shaders with EAT, and the groups inoculated with TAE and treated, the Log-rank test was used. For all groups, it was considered statistically significant when $\mathrm{P}<0.05$.

\section{Results and discussion}

\section{Target prediction}

For the target identification the PharmMapper server was used. The PharmMapper server is an open access web server designed to identify potential target candidates for the given small molecules (drugs, natural products or other newly discovered compounds with unidentified binding targets) using the pharmacophore mapping approach. PharmMapper hosts a large internal repertoire of pharmacophore database (ie PharmTargetDB) annotated from all target information in TargetBank, BindingDB, DrugBank and potential drug target database, including more than 7,000 pharmacophore models receptor-based (covering more than 1500 drug target information). The PharmMapper server classifies potential pharmacophoric groups based on their adjustment. The five main targets determined in this study are shown in the Table 1.

The target that presents the greatest possibility of fitting was the proto-oncogene serine/threonine-protein kinase Pim-1, which presents three hydrophobic groups for interaction (Figure 1). Pim-1 is directly involved in regulating cell cycle progression and apoptosis, and has been implicated in several types of cancer. In addition, Pim-1 is mainly involved in cytokine signaling and has been implicated in many signal transduction pathways, and its production is regulated by cytokines that regulate the STAT pathway, or STAT factors. These include interleukins, prolactin, TNF $\alpha$, EGF and IFN $\gamma$, among others. It is important to note that not only target Proto-oncogene serine/ threonine-protein kinase Pim-1 is related as a potential antitumor 
target, but MAP kinase-activated protein kinase 2, has already been studied as a possible target for the action of anticarcinogenic substances. ${ }^{30}$ The other potential targets found for compounds 1 , are related to diseases such as cardiomyopathy ${ }^{31}$ and inflammatory diseases. $^{32}$
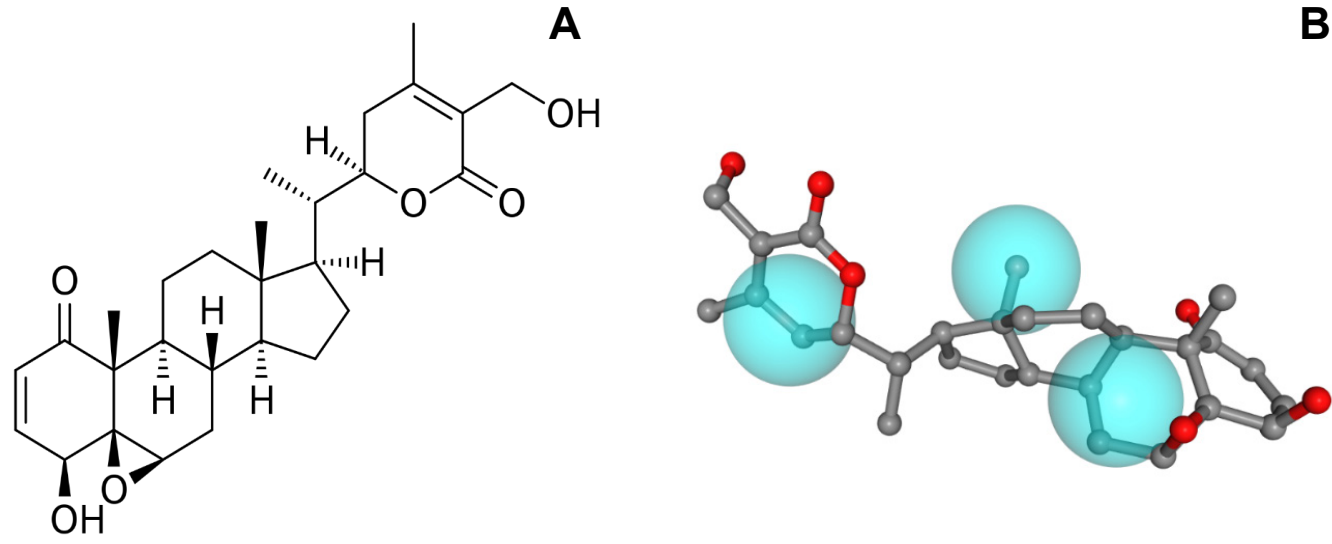

Figure I Chemical structure of Withaferin-A (I) (A).Alignment of compound I and the pharmacophore model of Proto-oncogene serine/threonine-protein kinase Pim-I (B).

Table I The five main potential targets related to Compound I predicted by PharmMapper

\begin{tabular}{llc}
\hline PDB ID & Target Name & Score \\
\hline 3BGP & $\begin{array}{l}\text { Proto-oncogene serine/threonine- } \\
\text { protein kinase PIMI }\end{array}$ & 2.99 I \\
2PIN & Thyroid hormone receptor beta & 2.989 \\
2ROY & Transthyretin & 2.986 \\
2P3G & MAP kinase-activated protein kinase 2 & 2.985 \\
IEUB & Collagenase 3 & 2.985 \\
\hline
\end{tabular}

\section{Pharmacokinetic properties}

The Swiss ADMET Predictor is a designed program of the computer for estimating pharmacokinetic parameters/properties of drug-like compounds from their molecular structures called the ADMET which referred to Absorption, Distribution, Metabolism, Excretion/Elimination, and Toxicity. ${ }^{33}$ We predicted the ADMET properties of compound $\mathbf{1}$ was performed using the free toolbar Swiss ADME (http://www.swissadme.ch/). ${ }^{29}$ Lipinski proposed four ADMET properties called the "Rule of Five". This rule of five was the authentic and "most well-known rule-based filter" of drug-likeness which is used to examine if the compound can be well absorbed orally or not. The rule of five includes; Molecular weight $(\mathrm{MW}) \leq 500$; Octanol/water partition coefficient (iLOGP) $\leq 5$; Number of hydrogen bond donors (HBDs) $\leq 5$ (accounted in function of $\mathrm{NH}$ or $\mathrm{OH}$ groups in the molecule); and Number of hydrogen bond acceptors (HBAs) $\leq 10$ (accounted in function of $\mathrm{N}$ or $\mathrm{O}$ atoms in the molecule). Under the Rule of Five, a molecule can only be orally active/absorb if it does not violate any two or more of the above rules. Table 2 represented some of the ADMET properties/parameters. ${ }^{34}$

According to the results obtained through the in silico approach, compound $\boldsymbol{I}$ obeys the standards of the Lipinski Rule, thus indicating a good oral bioavailability during the administration of this substance, when in an oral pharmaceutical form. The TPSA values obtained for the compound are below $140 \AA^{2}$, indicating an excellent permeability of the compound in the cellular plasma membrane. The percentage of absorption showed a result for the compound, with $75.75 \%$. The $\operatorname{LogS}$ of many substances already approved for use in therapeutics have a value greater than -4.00 . As shown in Table $1, \operatorname{LogS}$ showed a value of -4.97 . From these results, compound 1 is considered to be moderately soluble. The score of compound $\boldsymbol{l}$ for the Drug Score was determined by combining records of similarity with already approved drugs, lipophilicity, solubility, molecular mass and toxicity risks in a single numeric value, which ranges from 0.0 to 1.0 and can be used to predict the global potential of a compound as a new drug candidate. The value obtained in this approach was 0.55 suggesting that these compounds have the potential to become new drug candidates.

\section{Molecular electrostatic potential map}

The Molecular Electrostatic Potential Map (MEP) is a classic tool in the analysis of chemical activity, especially in the drug designer. In most cases, MEP is used as a qualitative approach. The MPE is based on the calculated properties of the charge density directly from the function of the molecular wave, and measures the interaction of a positively charged point with the nuclei and electrons of a molecule. The interaction between the molecules occurs between regions of opposite electrostatic potentials. Figure 2 shows the MEP of compound 1 , in two positions, where the blue colored areas suggest the potential for positive values, that is, electron deficiency, while the shades of the colored areas in red indicate negative values of the electrostatic potential, that is, electron-rich regions.

\section{Biological evaluation}

\section{Effects of $\boldsymbol{W}$. somnifera extract on the number of CFU-GM}

The effects of the administration of $W$. somnifera extract for 10 consecutive days, at doses of 20,50 , or $100 \mathrm{mg} / \mathrm{kg}$, on the number 
of granulocytes-macrophages of the bone marrow and spleen (CFU$\mathrm{GM}$ ) of the bone marrow of normal animals inoculated with EAT are shown in Figure 3A. In normal animals, the dose of $100 \mathrm{mg} / \mathrm{kg}$ increased the number of CFU-GM bone marrow ( $\mathrm{P}<0.01$, ANOVA, Tukey test) without producing changes in the number of splenic colonies. In animals with EAT (control), myelosuppression induced by this tumor model and expressed through a drastic reduction in the number of CFU-GM ( $\mathrm{P}<0.01$, ANOVA, Tukey Test) was restored to average values after treatment with doses of 20 and $50 \mathrm{mg} / \mathrm{kg}$ of $W$. somnifera Figure 3(B). A more significant number of colonies about the control was produced at a dose of $100 \mathrm{mg} / \mathrm{kg}(\mathrm{P}<0.001$, ANOVA, Tukey test). Concerning extramedullary hematopoiesis Figure 3(C). Then, it produced a significant increase in the number of CFU-GM of the spleen, when compared to the control group $(\mathrm{P}<0.001$, ANOVA, extramedullary Tukey test). The treatment of these animals with W.S. significantly reduced the number of splenic colonies in all groups evaluated $(\mathrm{P}<0.001$, ANOVA, Tukey Test), Figure 3C. However, the dose of $20 \mathrm{mg} / \mathrm{kg}$ of the group inoculated with TAE produced a significant reduction in the number of spleen colonies when compared to the group only inoculated with EAT, but a significant increase in the number of colonies when compared to the control group ( $\mathrm{p}<0.001$, ANOVA, Tukey test). Besides, in animals inoculated with TAE and treated with $100 \mathrm{mg} / \mathrm{kg} \mathrm{W}$. somnifera, the appearance of the colonies produced is similar to the colony produced by normal animals, with a dense nucleus and larger.
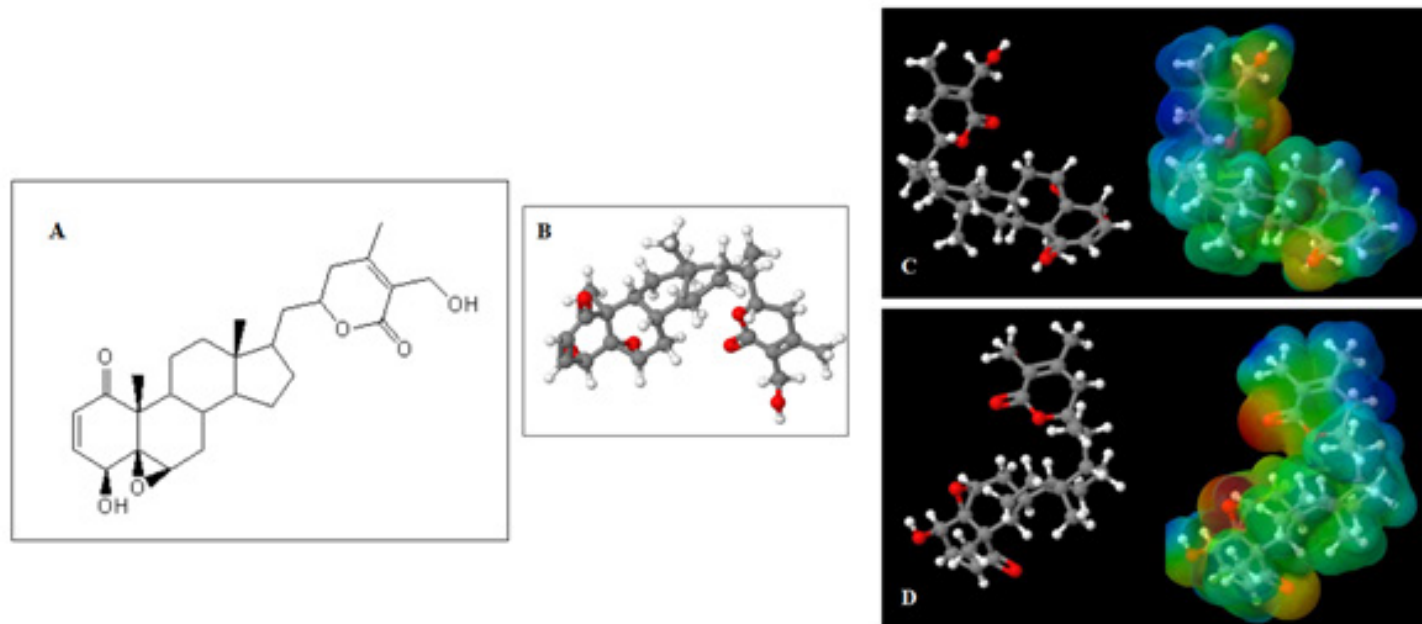

Figure 2 Overview of the structures of compound I.(A) 2D general structure of compound I; (B) 3D optimized structure of compound I; (C) Pose I and MEP of compound $I ;(D)$ Pose 2 and MEP of compound $I$.Although $(\boldsymbol{C})$ and $(\boldsymbol{D})$ are qualitatively similar to the surfaces of the MEP, there are significant differences in relation to the surface to the oxygen present in the epoxide group and in the ester group.

Table 2 ADMET parameters found for the compound I using the free toolbar SwissADMET Predictor

\begin{tabular}{llllllllllll}
\hline Compound & \multicolumn{1}{l}{ Lipinski parameter } & & & & TPSA $\left(\mathbf{A}^{2}\right)$ & LogS & Class & \%ABS (\%) & $\begin{array}{l}\text { Drug } \\
\text { Score }\end{array}$ \\
\hline & MW (g/mol) & HBDs & HBAs & iLOGP & $\mathrm{nV}$ & & & & & \\
$\mathrm{I}$ & 470.6 & 2 & 6 & 3.47 & 0 & 96.36 & -4.97 & moderately & 75.75 & 0.55 \\
\hline
\end{tabular}

Physicochemical properties calculated on Swiss ADME: MW, molecular weight; HBDs, hydrogen bonding donor; HBAs, hydrogen bonding acceptor; iLogP, octanol/water partition coefficient; $n V$, number of violations; TPSA, total polar surface area; LogS, coefficient of solubility determined by the ESOL method; Class: insoluble $<-10<$ poor $<-6<$ moderately $<-4<$ soluble $<-2<$ very $<0<$ highly; \%ABS was expressed by the equation $\%$ ABS $=109-(0.345 \times$ TPSA $)$.

\section{Effect of W. somnifera extract on colony-stimulating factor production (CSFs)}

The evaluation of the presence of colony-stimulating factors (CSFs) in the serum of normal animals showed a small colony-stimulating activity (Figure 3C). However, treatment with different doses (20, 50 , and $100 \mathrm{mg} / \mathrm{kg}$ ) of $W$. somnifera extract produced an increase in these factors, since their concentration increased significantly to normal animals ( $p<0.001-$ ANOVA, Tukey test). This increase in the concentration of colony-stimulating factors to the control group was even more evident in the group of animals only inoculated with EAT $(\mathrm{p}<0.001-$ ANOVA, Tukey Test). In the groups inoculated with EAT and treated with 3 doses $(20,50$, and $100 \mathrm{mg} / \mathrm{kg})$ of $W$. somnifera, the production of colony-stimulating factors was similar to the group only inoculated with $(\mathrm{p}<0.001-$ ANOVA, Tukey test $)$.

Effects of $W$. somnifera extract on the proliferative response of splenic cells

The effects of the administration of 20,50 , and $100 \mathrm{mg} / \mathrm{kg}$ of $W$. somnifera for 10 consecutive days on the blast transformation of lymphocytes from the spleen of animals inoculated with EAT are shown Figure 3D. A statistically significant inhibition $(\mathrm{p}<0.001-$ ANOVA - Tukey test) in the proliferative response of splenic cells to 
concanavalin A $(5 \mu \mathrm{g} / \mathrm{mL})$ was obtained for tumor-carrying animals However, a reversal of this effect was observed in animals treated with 50 and $100 \mathrm{mg} / \mathrm{kg}$ of $W$. somnifera, whose response to the stimulus was higher than that of differences, were lymphocytes of normal mice at a dose of $100 \mathrm{mg} / \mathrm{kg}$. On the other hand, in this assay, no observed in the functional activity of lymphocytes from normal mice treated with a dose of $20 \mathrm{mg} / \mathrm{kg}$ of plant extract (Figure 3D). The animals inoculated with EAT and treated with doses of 50 and $100 \mathrm{mg} / \mathrm{kg}$ of $W$. somnifera showed a significant increase in the proliferation of these cells when compared to the group only inoculated with TAE $(\mathrm{p}<0.001$ -ANOVA-Tukey Test).
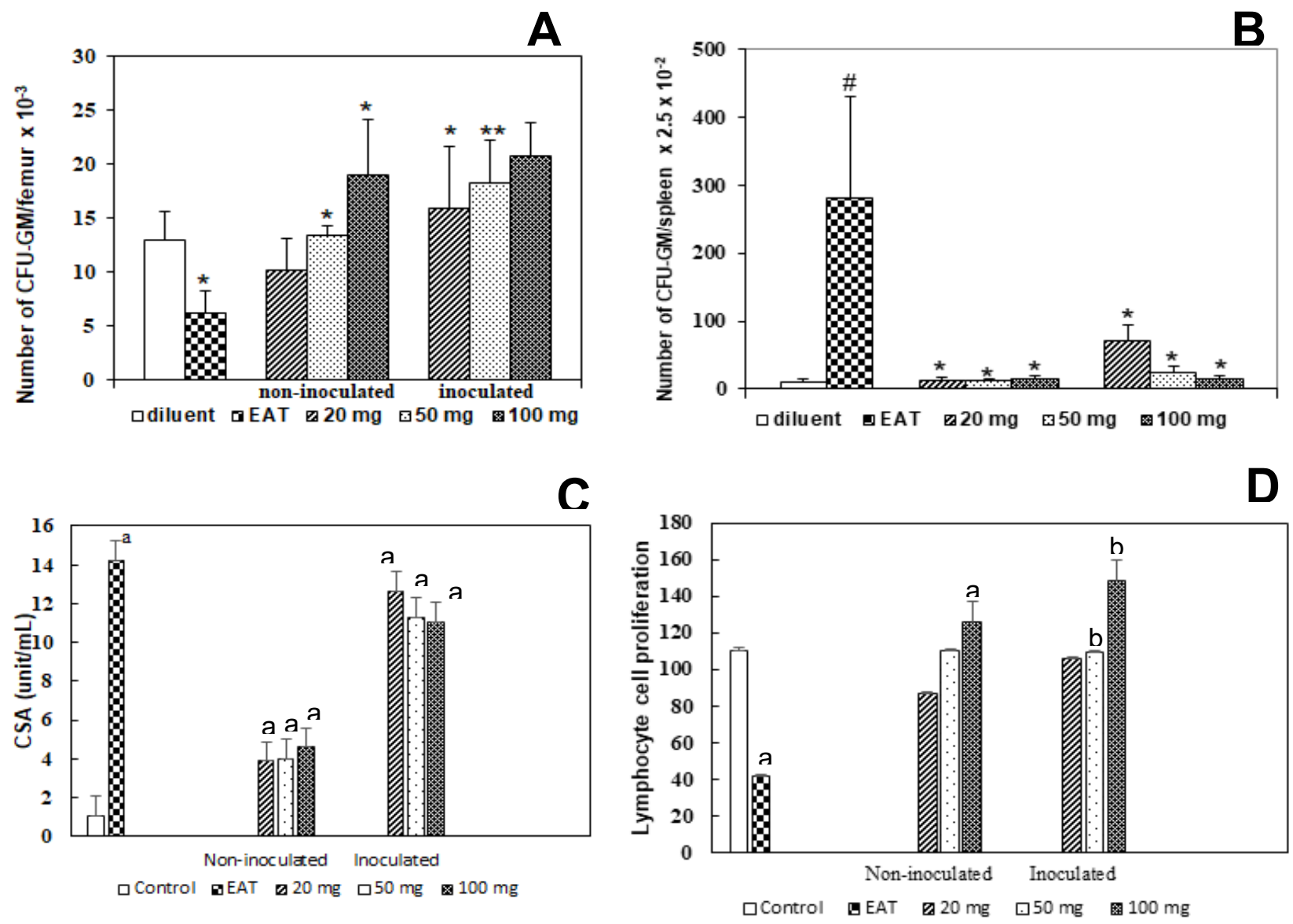

Figure 3 Mice treated with different doses (i.o.) of W. somnifera $\left(20-100 \mathrm{mg} / \mathrm{Kg}\right.$ ) for 10 days after inoculation of $6 \times 10^{6}$ Ehrlich ascites tumor cells, determined on the II days after tumor inoculation. Control mice received diluent only: (A) CFU-GM (femur). (B) Spleen granulocyte-macrophage colony units (CFU-GM). (C) Effects of serum of animals inoculated with EAT $\left(6 \times 10^{6}\right.$ cells/animal) and treated with different doses of W. somnifera extract $(20,50$, and $100 \mathrm{mg} / \mathrm{kg})$ for 10 consecutive days, directly on the bone marrow progenitor cell culture of normal animals. The animals were sacrificed $24 \mathrm{~h}$ after the end of treatment. (D) Proliferative response of splenic cells of animals inoculated with EAT $\left(6 \times 10^{6}\right.$ cells/animal) and treated with different doses of W. somnifera extract (20,50, and $100 \mathrm{mg} / \mathrm{kg}$ ) for 10 consecutive days. The animals were sacrificed $24 \mathrm{~h}$ after the end of treatment. The control groups received only drug diluent. Results represent the means \pm S.D. for eight mice.

(A) $* P<0.00$ I compared with the control group; $\# P<0.00$ I compared with control and tumor groups; $* * P<0.00$ I compared with tumor group. (B) \# $P<0.00$ I compared with the control group; ${ }^{*} P<0.00 \mathrm{I}$ compared with the tumor group. (C) ANOVA, Tukey Test. ${ }^{\mathrm{a}} P<0.00 \mathrm{I}$ compared to control; ${ }^{\mathrm{b} P}<0.00 \mathrm{I}$ compared to the group inoculated with EAT. (D) ANOVA, Tukey Test. ${ }^{a} P<0.00$ I compared to control; $P<<0.00$ I compared to inoculated with TAE.

Effects of W. somnifera extract on levels of cytokines TNF- $\alpha$ and IFN- $\gamma$

Cytokine production was evaluated in animals inoculated and treated with different concentrations of W.somnifera $(20,50$, or 100 $\mathrm{mg} / \mathrm{kg}$ ) for 10 days. The evaluations were performed in the splenic cell cultures for 24 and $48 \mathrm{~h}$ and the results are shown in Figure 2A and 2B. The levels of the TNF- $\alpha$ cytokine in the antinatant of cell cultures from animals only inoculated with EAT were not altered within $24 \mathrm{~h}$, while in the $48 \mathrm{~h}$, there was a significant reduction to the control group $(\mathrm{p}<0.001-$ Kruskal-Wallis ANOVA and Wilcoxon posteriori test) (Figures 4C and 4D). TNF- $\alpha$ levels in animals treated at a dose of $100 \mathrm{mg} / \mathrm{kg}$ were elevated to control, $24 \mathrm{~h}$ after incubation of cells, while with 20 and $50 \mathrm{mg} / \mathrm{kg}$, there was no change. Similarly, in $48 \mathrm{~h}$, we could observe high levels of this cytokine at doses of 50 and $100 \mathrm{mg} / \mathrm{kg}$. The antinatant of animals inoculated with TAE and treated with the dose of $100 \mathrm{mg} / \mathrm{kg} \mathrm{W}$. somnifera, collected after $24 \mathrm{~h}$ of culture, showed a significant increase of this cytokine to the control groups and only inoculated with the tumor. However, $48 \mathrm{~h}$ after incubation, all groups inoculated with EAT and treated with the 3 doses of $W$. somnifera revealed an increase in TNF- $\alpha$ compared to the only inoculated group Figure 4(A \& B). 
$\Delta$
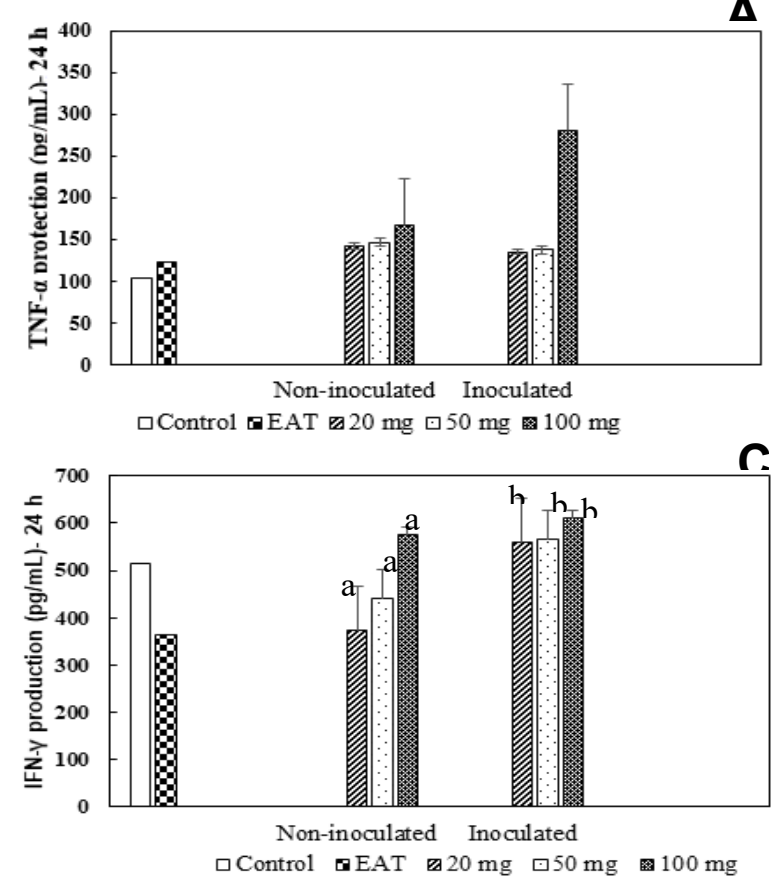

$\mathbf{R}$
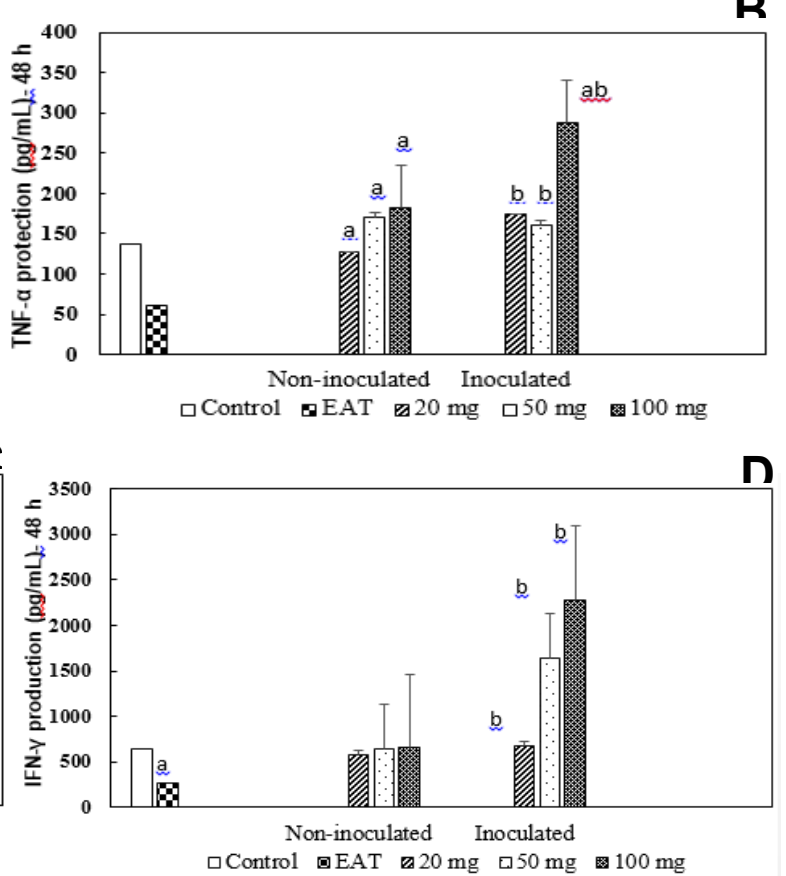

Figure 4 Parameters determined in the extranatant of splenic cell cultures of animals inoculated with EAT $\left(6 \times 10^{6}\right.$ cells/animal) and treated with different doses of Withania somnifera extract $(20,50$, and $100 \mathrm{mg} / \mathrm{kg})$ for 10 consecutive days. The animals were sacrificed $24 \mathrm{~h}$ after the end of treatment. The control groups received only drug diluent. (A) TNF- $\alpha$ concentration. The culture supernatant was collected $24 \mathrm{~h}$ after incubation. (B) TNF- $\alpha$ concentration. The culture supernatant was collected $48 \mathrm{~h}$ after incubation. (C) Concentration of INF- $\gamma$ determined in the antinatant. The culture supernatants were collected $24 \mathrm{~h}$ after incubation. (D) Concentration of INF- $\gamma$ determined in the antinatant. The culture supernatants were collected $48 \mathrm{~h}$ after incubation.

(A and B) Kruskal-Wallis ANOVA and Wilcoxon posterior test. ${ }^{a} P<0.0$ I compared to control; ${ }^{b} P<0.00$ I compared to the group inoculated with EAT. (C and D) Kruskal-Wallis ANOVA and Wilcoxon, a posteriori test. ${ }^{a} P<0.001$ compared to control; ${ }^{b} P<0.00$ I compared to TAE inoculated.

We also observed a significant reduction in the IFN- $\gamma$ levels in animals only inoculated with EAT at 24 and $48 \mathrm{~h}$ of incubation. On the other hand, there was a reversal condition in animals treated with doses of 20,50 , and $100 \mathrm{mg} / \mathrm{kg}$, in the periods of 24 and $48 \mathrm{~h}$ (Figure 4C and 4D). Animals treated with 20 and $50 \mathrm{mg} / \mathrm{kg}, 24 \mathrm{~h}$, a reduction in the IFN- $\gamma$ was observed, while an increase in the level of this cytokine with $100 \mathrm{mg} / \mathrm{kg}$ was detected. In $48 \mathrm{~h}$, no alterations were observed in the animals treated with the 3 doses of the plant. Briefly, we can conclude that the dose of $100 \mathrm{mg} / \mathrm{kg}$ of $W$. somnifera promotes an increase in the production levels of cytokine IFN- $\gamma$ and pro-inflammatory cytokines TNF- $\alpha$, being more effective in the two periods ( 24 and $48 \mathrm{~h}$ ) of incubation of splenic cells.

\section{Survival of animals inoculated with EAT and treated with W.} somnifera

The survival of animals inoculated with TAE and treated with 20 , 50 , or $100 \mathrm{mg} / \mathrm{kg}$ of $W$. somnifera extract for 10 days are shown in Figure 5. All animals with untreated tumors died until the 20 th day of observation, while treatment with 20,50 , and $100 \mathrm{mg} / \mathrm{kg}$ of W.S. significantly increased $(\mathrm{P}<0.01)$ the survival of these animals to 30,35 , and 40 days, respectively. The properties predicted by compound 1 , in theoretical studies on its pharmacokinetic properties, satisfy Lipinski's rule of five for oral bioavailability. In addition, the calculation of its electrostatic surface, shows significant load distribution differences, and it is possible to observe the regions with high and low electronic deficiency highlighted. Knowing that the active substance-receptor interactions happen, due to the presence of opposite charges in both ligands, this compound shows great potential for interaction. The results obtained with the prediction of biological targets revealed that compound 1 has great potential to be used as a substance for the treatment of tumor cells, since most of the targets found are related to the development of this disease, which is currently being treated at learn from the use of various drugs with a high degree of toxicity, through chemotherapy.

One of the significant side effects of chemotherapy is the damage to the immune system. The use of plants as the source of immunomodulatory material is still in its infancy. Some of the plants with known immunomodulatory activities are the Viscum album; ${ }^{27-29}$

Panax ginseng, Tinospora cordifolia, and others have been used in the cancer treatment. ${ }^{35-38}$ Polysaccharides, lectins, ${ }^{39}$ proteins, and peptide ${ }^{28}$ present in plants has been identified to stimulate the immune system. The present study was carried out mainly to determine the immunomodulatory activities of $W$. somnifera, an essential plant in Indian traditional medicine, to evaluate it as an adjuvant during chemotherapy. Thereby, in this work, we observed that the number of CFU-GM in the bone marrow of mice bearing the Ehrlich ascites tumor was reduced, whereas the number of CFU-GM in the spleen was increased. The administration of $W$. somnifera in tumor-bearing mice at doses varying from 20 to $100 \mathrm{mg} / \mathrm{kg}$ led to the stimulation of myelopoiesis. The optimal biologically active dose of $W$. somnifera $(100 \mathrm{mg} / \mathrm{kg})$, given 10 days after tumor inoculation, increased the number of CFU-GM in the bone marrow. Similarly, the dose of 100 
$\mathrm{mg} / \mathrm{kg}$ of $W$. somnifera also stimulated myelopoiesis in the bone marrow of normal mice. Conversely, this extract affected the number of CFU-GM in the spleen and reduced the number of CFU-GM in tumor-bearing mice at all doses used $(20,50$, and $100 \mathrm{mg} / \mathrm{Kg})$.

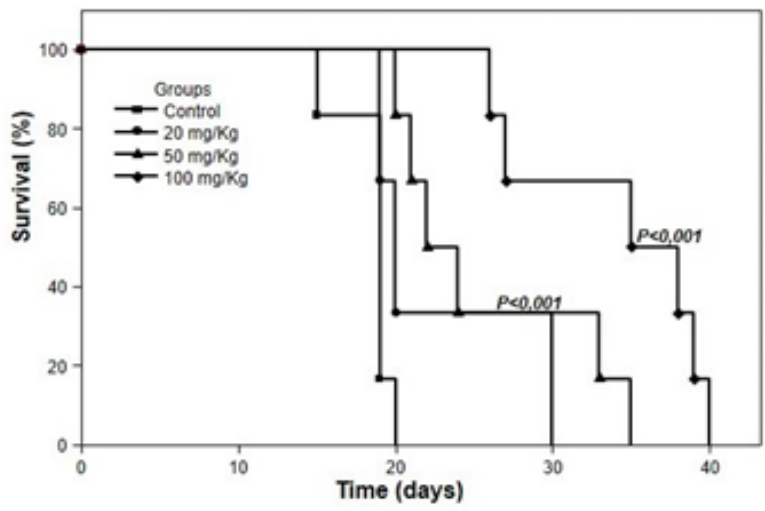

Figure 5 Survival of animals inoculated with Ehrlich tumor and treated with W. somnifera. The animals were treated at doses of 20,50 , and $100 \mathrm{mg} / \mathrm{kg}$ orally (gavage) for 10 days after intraperitoneal inoculation of $6 \times 10^{6}$ cells/ animal. The control animals received only diluent. Groups of 24 animals were observed daily for 30 days. $\mathrm{P}<0.0 \mathrm{I}$, compared to the tumor group (control) (Kaplan-Meier, Log-rank).

A variety of mechanisms could be postulated to explain the alterations produced in the CFU-GM compartment. The myeloproliferative response is dependent on several factors produced by many immunological cells, such as macrophage and lymphocytes. ${ }^{11,13,15,20,40,41}$ In this regard, it has been suggested ${ }^{42}$ that the myelosuppression observed in Ehrlich tumor-bearing animals is due to the production of inhibitory factors by macrophages. In our study, the effects of tumor load on myelopoiesis provide additional evidence to the documented ability of the Ehrlich ascites tumor to affect the receptor system of granulocyte-macrophage progenitor cells and a number of the functional capabilities of macrophages. ${ }^{11,16,42-45}$ Stimulation of myelopoiesis in the spleen of Ehrlich ascites tumorbearing mice is secondary to the tumor carriage in malignant ascites is the aggressive behavior of tumor-derived factor that stimulate the proliferation of splenic macrophages with suppressive activity. ${ }^{8,42-47}$ In this respect, Tomida et al. ${ }^{47}$ have reported that Ehrlich tumor cells produce factors capable of inducing proliferation of spleen cells with phenotypic and functional changes.

Evidence points to the role of inhibitory factors such as prostaglandin $\mathrm{E}_{2}$ and cytokines produced by macrophages and the tumor itself in the myelosuppression of tumor-bearing mice. ${ }^{48-50}$ Fecchio et al. ${ }^{16}$ have demonstrated that the intraperitoneal inoculation of Ehrlich ascites tumor cells induced the release of increased levels of prostaglandin $\mathrm{E}_{2}$ and a delayed inflammatory response. Increased macrophage production of prostaglandin $\mathrm{E}_{2}$ down-regulates $\mathrm{T}$ cell proliferation and blocks tumor necrosis factor- $\alpha$ stimulatory action on macrophages..$^{51,52}$ Additionally, it has been demonstrated that prostaglandin $\mathrm{E}_{2}$ favors $\mathrm{T}$ helper 2-like cytokine secretion profiles in murine and human $\mathrm{T}$ cells by inhibitory the production of $\mathrm{T}$ helper 1 -associated cytokines interleukin- 2 and interferon- $\gamma .{ }^{29,33,34,50,51,53-56} \mathrm{In}$ this respect, we observed that the dose of $100 \mathrm{mg} / \mathrm{kg}$ of W.somnifera promotes an increase in the production levels of cytokine IFN- $\gamma$ and pro-inflammatory cytokines TNF- $\alpha$, being more effective in the two periods ( 24 and $48 \mathrm{~h}$ ) of incubation of splenic cells.

It has been demonstrated that $W$. somnifera stimulates macrophagederived nitric oxide production through NF- $\mathrm{KB}$ transactivation in murine macrophages. ${ }^{3}$ The authors suggested that this effect could increase the macrophage cytotoxic/cytostatic response and provide a mechanistic basis for the immunostimulating properties of $W$. somnifera. Moreover, Dhuley ${ }^{5}$ demonstrated that this plant stimulates chemotaxis and the production of Interleukin-1 and TNF- $\alpha$ in macrophages from immunosuppressed mice. The in vitro addition of serum from treated mice to cultures of normal myelopoietic cells produced a stimulating effect on the growth and differentiation of these cells, suggesting that the stimulatory effects observed on the myelopoiesis in response to $W$. somnifera extract could be due to a functionally relevant increase in the levels of the colony-stimulating factor. In parallel, we demonstrated that the treatment with $W$. somnifera before a lethal dose of the bacteria significantly prolonged the survival of these animals, with survival rates of up to $70 \%$.

Our present study showed that the dose of $100 \mathrm{mg} / \mathrm{kg} \mathrm{W}$. somnifera elicited optimal modulatory effects on bone marrow and spleen colony formation and increased the survival mice. These results suggest that the antitumor activity of $W$. somnifera might be related to some ability of this extract in keeping the balance between positive and negative stimulus controlling myelopoiesis, which would be expected to result from changes in the local behavior of various cell types, particularly $\mathrm{T}$ cells and macrophages. Therefore, it is very likely that the antitumor effect of $W$. somnifera, acting synergistically with other factors, such as specific cytokines, may further result from enhanced macrophage activation towards Ehrlich ascites tumor cells entirely. In the line of this suggestion, the reduction in the number of CFU-GM in the spleen of treated tumor-bearing mice could be ascribed in part to a decreased production of tumor-derived factors responsible for the disturbed hematopoietic activity in this organ. The results obtained in in vitro studies corroborate with the results predicted in the determination of biological targets since the use of $W$. somnifera extract mainly at the dose of $100 \mathrm{mg} / \mathrm{kg}$, helped increase the levels of cytokine production IFN- $\gamma$ and pro-inflammatory cytokines TNF- $\alpha$, a result that may be related to the interaction of compound 1 with the enzyme PIM1. These findings are particularly important in the context to consider $W$. somnifera for combination chemotherapy to protect the host from hematotoxicity as well as to supplement the tumoricidal efficacy.

\section{Acknowledgments}

This study was supported by Unicamp, Unipinhal, UNESP, Instituto de Ciências e Tecnologia and Centro Universitário FUNVIC.

\section{Conflicts of interest}

The author declares that there is no conflict of interest.

\section{References}

1. Winters M. Ancient medicine, modern use: Withania somnifera and its potential role in integrative oncology. Altern Med Review. 2006;11(4):269-277.

2. Dar NJ, Ahmad M. Neurodegenerative diseases and Withania somnifera (L.): An update. J Ethnopharmacol. 2020;256:112769. 
3. Singh N, Bhalla M, De Jager P. et al. An overview on ashwagandha: a rasayana (rejuvenator) of Ayurveda. African J Tradit Complement Altern Med. 2011;8(S):208-213.

4. Chaudhary SP. An Ayurvedic review of ashwagandha from samhitha and nighantus. Experiential study of shilajatu formulation on madhumeha. View project an Ayurvedic Review of Ashwagandha from Samhitha and Nighantus. Prakash. World J of Pharm Res. 2015;4(10):2736-2745.

5. Dhuley JN. Effect of some Indian herbs on macrophage functions in ochratoxin A treated mice. J Ethnopharmacol. 1997;58(1):15-20.

6. Shohat B, Gitter S, Lave D. Effect of withaferin on Ehrlich ascites tumor cells - cytological observations. Int J Cancer. 1970;5:244-252.

7. Balasubramanyam M, Venkateswaran A, Mohan V. Whithania somnifera (herbal extract) inhibits cellular store-operated $\mathrm{Ca}^{2+}$ entry in patients with type 2 diabetes. Diabetes Res Clin Pract. 2000;50:376377.

8. Watson GA, Fu Y-X, Lopez DM. Splenic macrophages from tumorbearing mice co- expressing MAC-1 and MAC-2 antigens exert immunoregulatory functions via two distinct mechanisms. J Leukoc Biol. 1991;49:126-138.

9. O'Connor MA, Rastad JL, Green WR. The role of myeloid-derived suppressor cells in viral infection. Viral Immunol. 2017;30:82-97.

10. Romano A, Parrinello NL. Regulatory myeloid cells in hematological malignancies. Haematologica. 2017;102:208-209.

11. Teixeira ST, Valadares MC, Gonçalves AS, et al. Prophylactic administration of Withania somnifera extract increases host resistance in Listeria monocytogenes infected mice. Int Immunopharmacol. 2006;6(10):1535-1542.

12. Melo A, Justo GZ, de Souza Queiroz ML. Stimulation of myelopoiesis in Listeria monocytogenes-infected mice by an aggregated polymer isolated from Aspergillus oryzae. Hum Exp Toxicol. 2001;20(1):38-45.

13. Justo GZ, Durán N, Queiroz MLS. Myelopoietic response in tumourbearing mice by an aggregated polymer isolated from Aspergillus oryzae. Eur J Pharmacol. 2000;388(3):219-226.

14. Hamilton JA. Colony-stimulating factors in inflammation and autoimmunity. Nat Rev Immunol. 2008:8(7):533-544.

15. De Souza Queiroz ML, Valadares MC, Bincoletto C, et al. Ehrlich ascites tumor as a tool in the development of compounds with immunomodulatory properties. Immunopharmacol Immunotoxicol. 2004;26(4):511-525.

16. Fecchio D, Sirois P, Russo M, et al. Studies on inflammatory response induced by Ehrlich tumor in mice peritoneal cavity. Inflammation. 1990;14(1):125-132.

17. Segura JA, Barbero LG, Márquez J. Early tumor effect on splenic lymphocytes in mice. FEBS Lett. 1997;414(1):1-6.

18. Valadares MC, Ramos AL, Rehmann FJK, et al. Antitumour activity of [1,2- di(cyclopentadienyl)-1,2-di(p-N,N- dimethylaminophenyl)ethanediyl] titanium dichloride in xenografted Ehrlich's ascites tumour. Eur J Pharmacol. 2006;534(1-3):264-270.

19. Fan G, Jiang X, Wu X, et al. Anti-Inflammatory Activity of Tanshinone IIA in LPS- Stimulated RAW264.7 Macrophages via miRNAs and TLR4-NF-кB Pathway. Inflammation. 2016;39(1):375-384.

20. Metcalf D. The colony-stimulating factors and cancer. Cancer Immunol Res. 2013;1(6):351-356.

21. Metcalf D. Hematopoietic cytokines. Blood. 2008;111(2):485-491.
22. Xie J, Itzkowitz SH. Cancer in inflammatory bowel disease. World $J$ Gastroenterol. 2008; 14(3):378-389.

23. Schroeder MA, Ritchey J, Di Persio JF. Myeloid suppressive cells mobilized by GM- CSF in non-tumor bearing mice are dependent on interferon gamma for function. Blood. 2012;120(21):832.

24. Sehgal N, Gupta A, Valli RK, et al. Withania somnifera reverses Alzheimer's disease pathology by enhancing low-density lipoprotein receptor-related protein in liver. Proc Natl Acad Sci USA. 2012;109:3510-3515.

25. Engh G, van den Bol S. The presence of a CSF enhancing activity in the serum of endotoxin-treated mice. Cell Prolif. 1975;8(6):579-587.

26. Yoon TJ, Yoo YC, Kang TB, et al. Lectins isolated from Korean mistletoe (Viscum album coloratum) induce apoptosis in tumor cells. Cancer Lett. 1999;136(1):33-40.

27. Kuttan G, Kuttan R. Immunomodulatory activity of a peptide isolated from Viscum album extract (NSC 635 089). Immunol Invest. 1992;21(4):285-296.

28. Sadananda T. In vitro antioxidant activity of lectin from different endophytic fungi of Viscum album L. Br J Pharm Res. 2014;4(5): 626-643.

29. Daina A, Michielin O, Zoete V. SwissADME: a free web tool to evaluate pharmacokinetics, drug-likeness and medicinal chemistry friendliness of small molecules. Sci Rep. 2017;7:42717.

30. Adhipandito CF, Ludji DPKS, Aprilianto E, et al. Matrix metalloproteinase 9 as the protein target in anti-breast cancer drug discovery: an approach by targeting hemopexin domain. Futur J Pharm Sci. 2019;5(1).

31. Hanna M. Novel drugs targeting transthyretin amyloidosis. Curr Heart Fail Rep. 2014;11(1):50-57.

32. Fiore M, Forli S, Manetti F. Targeting Mitogen-Activated Protein Kinase-Activated Protein Kinase 2 (MAPKAPK2, MK2): Medicinal Chemistry Efforts To Lead Small Molecule Inhibitors to Clinical Trials. J Med Chem. 2016;59(8):3609-3634.

33. Singh DB, Gupta MK, Singh DV, et al. Docking and in silico ADMET studies of noraristeromycin, curcumin and its derivatives with Plasmodium falciparum SAH hydrolase: a molecular drug target against malaria. Interdiscipl Sci Comput Life Sci. 2013;5(1):1-12.

34. Lipinski CA, Lombardo F, Dominy BW, et al. Experimental and computational approaches to estimate solubility and permeability in drug discovery and development settings. Adv Drug Deliv Rev. 1997;23(1-3):3-25.

35. Mancuso C, Santangelo R. Panax ginseng and Panax quinquefolius: from pharmacology to toxicology. Food Chem Toxicol. 2017;107(Pt A):362-372.

36. Yadav RNS, Agarwala M. Phytochemical analysis of some medicinal plants. J Phytol. 2011;3(12):10-14.

37. Singh K, Panghal M, Kadyan S, et al. Antibacterial activity of synthesized silver nanoparticles from Tinospora cordifolia against multi drug resistant strains of Pseudomonas aeruginosa isolated from burn patients. J Nanomed Nanotechnol. 2014;5(2):192.

38. Kuttan G. Use of Withania somnifera Dunal as an adjuvant during radiation therapy. Indian J Exp Biol. 1996;34(9):854-856.

39. Queiroz MLS, Justo GZ, Valadares MC, et al. Evaluation of Caesalpinia ferrea extract on bone marrow hematopoiesis in the murine models of listeriosis and Ehrlich ascites tumor. Immunopharmacol Immunotoxicol. 2001;23(3):367-382. 
40. Queiroz MLS, Justo GZ, Valadares MC, et al. Adjuvant effect of Pluchea quitoc extract on the resistance of tumor-bearing mice by modulation of the host hematopoietic response. Immunopharmacol Immunotoxicol. $2001 ; 23(2): 215-228$.

41. Pessina A, Brambilla P, Villa S, et al. CFU-s and CFU-c Proliferation after treatment of normal bone marrow cells with Ehrlich ascitic fluid. Oncology. 1982;39:391-395.

42. Keeb G, Lala PK. Effects of Ehrlich ascites tumor transplantation in mice on the distribution of cells capable of forming hemopoietic colonies in vitro. Eur J Cancer. 1978;14(4):331-335.

43. Pessina A. Brambilla P. Villa S, et al. Factors produced by macrophages and tumor cells: influence on the granulocytic precursor cells (CFU-C) in normal and tumor-bearing mice. $J$ Cancer Res Clin Oncol. $1982 ; 102: 235-244$

44. Justo GZ, Durán N, Queiroz MLS. Natural killer cell activity, lymphocyte proliferation, and cytokine profile in tumor-bearing mice treated with MAPA, a magnesium aggregated polymer from Aspergillus oryzae. Immunopharmacol Immunotoxicol. 2003;25(3):305-319.

45. Hardy CL, Balducci L. Review: hemopoietic alterations of cancer American Journal of the Medical Sciences. 1985;290(5):196-205.

46. Kobari L, Weil D, Lemoine FM, et al. Secretion of tumor necrosis factor-alpha by fresh human acute nonlymphoblastic leukemic cells: role in the disappearance of normal CFU- GM progenitors. Exp Hematol. 1990;18(11):1187-1192.

47. Tomida M, Yamamoto-Yamaguchi Y, Hozumi M. Purification of a factor inducing differentiation of mouse myeloid leukemic M1 cells from conditioned medium of mouse fibroblast L929 cells. J Biol Chem. 1984;259(17):10978-10982.
48. Bouffi C, Bony C, Courties G, et al. IL-6-dependent PGE2 secretion by mesenchymal stem cells inhibits local inflammation in experimental arthritis. PLoS One. 2010; 5(12):e14247.

49. Koul B. Herbs for cancer treatment. Singapore: Springer Nature eBook, 2020

50. Alleva DG, Burger CJ, Elgert KD. Interferon- $\gamma$ reduces tumor-induced Ia- macrophage- mediated suppression: role of prostaglandin E2, Ia, and tumor necrosis factor- $\alpha$. Immunopharmacol. 1993;25(3):215-227.

51. Elgert KD, Alleva DG, Mullins DW. Tumor-induced immune dysfunction: the macrophage connection. J. Leukocyte Biol. 1998;64(3):275-290.

52. Sobhani M, Farzaei MH, Kiani S, et al. Immunomodulatory, antiinflammatory/antioxidant effects of polyphenols: a comparative review on the parental compounds and their metabolites. Food Rev Int. 2020;153.

53. Packard KA, Khan MM. Effects of histamine on Th1/Th2 cytokine balance. Int Immunopharmacol. 2003;3(7):909-920.

54. Kawahara K, Hohjoh H, Inazumi T, et al. Prostaglandin E2-induced inflammation: Relevance of prostaglandin E receptors. Biochim Biophys Acta. 2015;1851(4):414-421.

55. Xiaofeng Liu, Sisheng Ouyang, Biao Yu, et al. PharmMapper Server: a web server for potential drug target identification via pharmacophore mapping approach. Nucleic Acids Res. 2010;38:W609-W614.

56. Xia W, Chenxu P, Jiayu G, et al. Enhancing the Enrichment of Pharmacophore-Based Target Prediction for the Polypharmacological Profiles of Drugs. J Chem Inf Model. 2016; 56:1175-1183. 